\title{
Moderne Senologie aus der Sicht der Gynäko-Onkologie
}

\section{G. Emons, Co-Kongresspräsident der 31. Jahrestagung der Deutschen Gesellschaft für Senologie}

In fast allen Ländern ist die Diagnostik, operative und systemische Therapie des Mammakarzinoms sowie die Nachsorge auf viele Fachdisziplinen verteilt. In Deutschland sind entsprechend qualifizierte Gynäkologinnen und Gynäkologen involviert in den Mammografie-Screening Programmen und der radiologischen und sonografischen Diagnostik inklusive der interventionellen Maßnahmen. Gynäkologinnen und Gynäkologen zeichnen verantwortlich für die operative und die Systemtherapie. Die primäre Nachsorge und auch die Betreuung von Frauen in der metastasierten Situation wird an vielen Orten ebenfalls primär durch Frauenärztinnen und -ärzte gewährleistet. Diese Konstellation bietet unseren Patientinnen den Vorteil, dass sie in der Diagnostik, Behandlung und Nachsorge ihres Mammakarzinoms so gut wie nie die Fachdisziplin wechseln müssen, sieht man einmal von der Strahlentherapie ab. So werden Missverständnisse und Übertragsfehler an den Schnittstellen minimiert. Daneben haben Gynäkologinnen und Gynäkologen eine große Kompetenz bezüglich der Ovarien und der Sexualsteroide, sodass Störungen des Endokriniums als Nebenwirkung einer Chemotherapie oder die beabsichtigten Folgen einer endokrinen Therapie ihnen eher geläufig sind als Kolleginnen und Kollegen anderer Fachgebiete.

Die Musterweiterbildungsordnung der Bundesärztekammer sowie die Weiterbildungsordnungen der Landesärztekammern tragen dieser Entwicklung Rechnung, indem sie umfangreiche Kenntnisse in der Diagnostik, Therapie und Nachsorge des Mammakarzinoms für die Weiterbildung im Schwerpunkt „gynäkologische Onkologie“ fordern.

Wir können heute klar sagen, dass eine Gynäkologin bzw. ein Gynäkologe mit dem erfolgreichen Abschluss dieser Schwerpunktweiterbildung eine hohe Kompetenz auf dem Gebiet des Mammakarzinoms besitzt.

Dennoch sollten wir bescheiden bleiben. In der Diagnostik sind wir ohne qualifizierte Radiologen und Nuklearmediziner sehr limitiert. Die Pathologen liefern zentrale Komponenten der Diagnosestellung und Therapieplanung. Bei aufwändigeren rekonstruktiven Operationen ist es oft sinnvoll, mit einem plastischen Chirurgen zu kooperieren. Die Strahlentherapie der Mamma ist Domäne der Radiotherapeuten. Und auch in der Systemtherapie ist die Kooperation mit internistischen Onkologen und Palliativmedizinern bei Multimorbidität und im fortgeschrittenen metastasierten Stadium meist segensreich.

Das heißt, trotz der zentralen Rolle, die die Gynäkologie in Deutschland in der Vorsorgung von Mammakarzinompatientin- nen spielt, ist eine enge Interaktion aller genannten Disziplinen erforderlich, wenn eine gute Qualität erreicht werden soll.

Garant für diese Interdisziplinarität ist die Deutsche Gesellschaft für Senologie, an deren Gründung wiederum weitsichtige Gynäkologen beteiligt waren. Der DGS ist es in den letzten 30 Jahren in hervorragender Weise gelungen, die verschiedenen Disziplinen zusammenzuführen und zusammenzuhalten. Auch wenn hier und dort Partikularinteressen zum Vorschein kommen, hat sich doch ein „senologisches Wir-Gefühl“ entwickelt, bei dem das Verbindende, d.h. die gemeinsame Verantwortung für Frauen mit Brustkrebs im Vordergrund steht.

Neben den erfolgreichen interdisziplinären Jahrestagungen der DGS hat sich der interdisziplinäre Gedanke der Senologie in vorbildlicher Weise in den zertifizierten Brustzentrum manifestiert. Hier wird Interdisziplinarität im Interesse unserer Patientinnen gelebt. Die externe Qualitätskontrolle ist zwar wichtig, aber im Vergleich zu der Etablierung des gemeinsamen, eng verzahnten Handelns diverser Fachabteilungen eher sekundär.

Auch in der Fachgesellschaft der Gynäkologischen Onkologen, der AGO, ist nicht zuletzt dankt der DGS das Prinzip der Interdisziplinarität fest etabliert. Die Kommission Mamma zählt stolz Radiologen, Strahlentherapeuten, internistische Onkologen und Pathologen zu ihren Mitgliedern. Die jährlich herausgegebenen Empfehlungen dieser Kommission werden interdisziplinär erarbeitet. Die Kooperation zwischen AGO und DGS ist vorbildlich durch gegenseitige Beteiligung an den Tagungen, sowie Repräsentanz in den jeweiligen Vorständen. Die Gynäkologische Onkologie dankt der DGS für 30Jahre gute und erfolgreiche Zusammenarbeit und wünscht beiden Gesellschaften eine gute gemeinsame Zukunft.

\section{Bibliografie}

DOI http://dx.doi.org/10.1055/s-0031-1271494

Senologie 2011; 8: 73

(c) Georg Thieme Verlag KG Stuttgart · New York · ISSN 1611-6453

\section{Korrespondenzadresse}

Prof. Dr. med. G. Emons

Ärztlicher Direktor der Universitäts-Frauenklinik

Universitätsklinikum Göttingen

Robert-Koch-Str. 40

37075 Göttingen

emons@med.uni-goettingen.de 